Nervenarzt 2021 · 92:1283-1292

https://doi.org/10.1007/s00115-021-01154-5

Angenommen: 10. Mai 2021

Online publiziert: 7. Juli 2021

(c) Der/die Autor(en) 2021

\section{Impfen und Multiple Sklerose}

Das Impfwesen gehört zu den größten Errungenschaften der Medizingeschichte. Begonnen hat alles in der Medizinhistorie des Abendlandes mit der Inokulation von Kuhpocken durch den englischen Arzt Edward Jenner 1796 [55]. Danach entdeckte Robert Koch erstmals Bakterien als Ursache für den Milzbrand und die Tuberkulose.

In weiterer Folge wurden die ersten Impfstoffe gegen Milzbrand und Tollwut mittels gezüchteter attenuierter Keime durch Louis Pasteur und Emile Roux entwickelt [80]. Diese enormen Fortschritte im Bereich der Vakzinologie setzten sich immer weiter fort, sodass wir inzwischen im Zeitalter der genbasierten Impfstoffe angelangt sind [57]. Die Wirksamkeit von Impfstoffen kann nicht zuletzt durch die Ausrottung der Pocken, beinahe Ausrottung von Poliomyelitis oder der massiven Eindämmung von Erkrankungen wie der Diphtherie, Masern, Mumps oder Röteln

Teil 1 dieses Beitrags Die Corona-Pandemie und Multiple Sklerose: Impfungen und deren Implikationen für Patienten - Teil 1: Empfehlungen finden Sie unter https://doi.org/10.1007/s00115021-01155-4.

Tobias Monschein · Tobias Zrzavy' · Micha Löbermann ${ }^{2}$ Alexander Winkelmann ${ }^{3}$. Thomas Berger ${ }^{1} \cdot$ Paulus Rommer ${ }^{1,5} \cdot$ Hans-Peter Hartung ${ }^{1,4} \cdot$ Uwe K. Zettl $^{5}$

${ }^{1}$ Universitätsklinik für Neurologie, Medizinische Universität Wien, Wien, Österreich

${ }^{2}$ Abteilung für Tropenmedizin und Infektionskrankheiten, Universitätsmedizin Rostock, Rostock, Deutschland

${ }^{3}$ Klinik und Poliklinik für Neurologie, Universitätsmedizin Rostock, Rostock, Deutschland

${ }^{4}$ Klinik für Neurologie, Universitätsklinikum Düsseldorf, Medizinische Fakultät, Heinrich-Heine-Universität, Düsseldorf, Deutschland

${ }^{5}$ Klinik und Poliklinik für Neurologie, Neuroimmunologische Sektion, Universitätsmedizin Rostock, Rostock, Deutschland

\title{
Die Corona-Pandemie und Multiple Sklerose: Impfungen und deren Implikationen für Patienten - Teil 2: Impfstofftechnologien
}

zweifellos belegt werden [49]. Das Ziel von Impfprogrammen ist daher nicht nur der Individualschutz, sondern auch die Elimination bzw. das Zurückdrängen von Erkrankungen sowie auch die Verhinderung von Epidemien und das Erlangen einer Herdenimmunität [80].

Impfungen gelten im Allgemeinen als sicher. In diesem Zusammenhang sollte zwischen Impfreaktionen, Impfnebenwirkungen und Impfschäden unterschieden werden [80]. Impfreaktionen sind häufig, harmlos und passagere Beschwerden, welche von Lokalreaktionen über grippeähnliche Symptome bis hin zu „Impfmasern“ im Falle der Masernimpfung reichen können [78]. Im Gegensatz dazu wird eine Impfnebenwirkung durch eine schädliche und unbeabsichtigte Reaktion auf eine Impfung definiert [78]. Der Begriff des Impfschadens leitet sich aus dem Infektionsschutzgesetz ab und ist definiert durch ,gesundheitliche und wirtschaftliche Folgen einer über das übliche Ausmaß hinausgehenden gesundheitlichen Schädigung durch eine Schutzimpfung" [78]. Insgesamt sind Impfnebenwirkungen und -schäden sehr selten [78]. In diesem Zusammenhang ist ein wichtiges Beispiel mit enormen
Folgen eine Publikation von Wakefield et al. im Fachjournal The Lancet aus dem Jahre 1998 [76]. Hier wurde anhand von nur 12 Kindern und ohne Kontrollgruppe ein Zusammenhang von Autismus und der Masern-Mumps-Röteln-Impfung (MMR) vermutet. Die Studie wurde 2010 zurückgezogen, weil sie falsifiziert wurde, resultierte jedoch in einem extrem relevanten Rückgang der Durchimpfungsraten und entsprechenden Zuwachs der Impfskepsis [80]. Mittlerweile konnte in zahlreichen Aufarbeitungen gezeigt werden, dass es keinen Zusammenhang zwischen der MMR-Impfung und Autismus gibt [70, 71].

Bezugnehmend auf Multiple Sklerose (MS) wurde beispielsweise ein erhöhtes MS-Risiko im Zusammenhang mit der Hepatitis-B-Impfung postuliert [25]. Dies bestätigte sich jedoch ebenfalls in einer großen Fall-Cross-over-Studie von Confavreux nicht [12]. Dem folgend konnte, trotz zahlreicher epidemiologischer Studien, bis heute keine Verursachung oder Auslösung von Multipler Sklerose durch Impfungen nachgewiesen werden, genauso wenig wie das Auslösen eines Schubes (außer in einigen Fallse- 


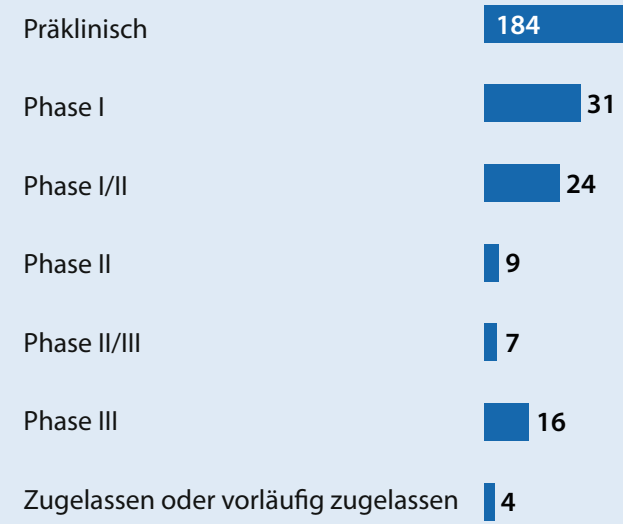

Zugelassen oder vorläufig zugelassen
Novel Coronavirus Landscape COVID-19 WHO
Abb. 1 Überblick über den Entwicklungsstand rezenter SARS-CoV-2-Impfstoffkandidaten (Stand 23.04.2021) rien zur Gelbfieberimpfung; [15, 35]). Bezüglich der Typhusimpfung wurde sogar ein potenziell positiver Einfluss auf den Erkrankungsverlauf der MS hypothetisiert [86]. Des Weiteren konnte gezeigt werden, dass Impfen mit einer geringeren Wahrscheinlichkeit verbunden ist, innerhalb der nächsten 5 Jahre an MS zu erkranken [23]. Da gerade für MSPatienten Infektionen ein erhebliches Risiko mit sich bringen, gilt es, gerade diese durch Impfungen zu schützen [16, 42, 86].

\section{COVID-19-Impfungen und MS}

Die insgesamt 275 SARS-Cov-2-Impfstoffe, die sich in der Entwicklung befinden, spiegeln den enormen wissenschaftlichen Fortschritt seit Anbeginn der Pandemie wider (Details siehe D Abb. 1; [108]).

Weltweit haben es bisher 14 Impfstoffe zur Zulassung geschafft [89]. Davon sind die beiden mRNA-Impfstoffe von BionTech/Pfizer (BNT162b2 am 21.12.2020) und Moderna (mRNA1273 am 06.01.2021) sowie der nichtreplizierende Vektorvirusimpfstoff von AstraZeneca (ChAdOx1 nCoV-19 am 29.01.2021) und jener von Johnson\&Johnson (Janssen COVID-19 Vaccine am 11.03.2021) durch die Europäische Arzneimittelbehörde (EMA) in der Europäischen Union (EU) zugelassen worden $[3,56,75,94]$. Des Weiteren wurden die nichtreplizierenden Vektorvirusimpfstoffe Sputnik-V (bereits in Phase II am 11.08.2020) in Russland, Covaxin (BBV152) in Indi- en (am 02.01.2021 ohne veröffentlichte Wirksamkeitsdaten), „Serum Institute of India - Covishield" in Indien (gleiche Formulierung wie AstraZeneca) sowie „CanSino: Ad5-nCoV“ in China zugelassen $[38,51,75,88]$. An Protein-SubunitImpfstoffen wurden „Anhui Zhifei Longcom - RBD-Dimer“ in China und „FBRI: EpiVacCorona" in Russland zugelassen $[52,82]$. Schließlich wurden 3 inaktivierte Totimpfstoffe („Sinopharm [Beijing] BBIBP-CorV“, „Sinopharm [Wuhan] Inactivated [Vero Cells] “ und „Sinovac CoronaVac“) in China, 1 („Bharat Biotech Covaxin") in Indien und 1 (Chumakov Center: KoviVac) in Russland zugelassen [81, 84, 87, 89].

Die Wirksamkeitsprofile der mRNAImpfstoffe sind mit $95 \%$ bei der Prävention von COVID-19 beim BionTech/ Pfizer-Impfstoff sowie $94,1 \%$ bei jenem von Moderna als hervorragend anzusehen. Auch das Sicherheitsprofil gilt als sehr gut. Die Nebenwirkungen waren im Wesentlichen auf lokale Reaktionen und milde systemische Reaktionen beschränkt. Beim BionTech/ Pfizer-Impfstoff wurden vier schwere Nebenwirkungen (SAEs) berichtet (Schulterverletzung im Zusammenhang mit der Impfstoffverabreichung, axilläre Lymphadenopathie rechts, paroxysmale ventrikuläre Arrhythmie und Parästhesie im rechten Bein; [56]). Im Falle des Moderna-Impfstoffs wurden Fazialisparesen dokumentiert. Diese traten jedoch in beiden Gruppen mit einer Häufigkeit von $<0,1 \%$ auf [3]. Des Weiteren wurden in der Phase-III-Zulassungsstudie des Moderna-Impfstoffs auch Risikogruppen, einschließlich immunsupprimierter Patienten mit HIVInfektion, eingeschlossen. Daran anschließend wurde für den BionTech/ Pfizer-Impfstoff ein Sicherheitsupdate nach der Zulassung veröffentlicht, das keine neuen Sicherheitsaspekte erbrachte [95].

Der Impfstoff von AstraZeneca weist in der gepoolten Analyse gegenüber den mRNA-Impfstoffen mit $70 \%$ eine niedrigere Wirksamkeit auf, wobei die Vergleichbarkeit nur bedingt gegeben ist. Das Sicherheitsprofil war insgesamt ebenfalls gut, dennoch wurden in der klinischen Phase-III-Studie 3 Fälle von transverser Myelitis (TM) dokumentiert [75]. Einem unabhängigen neurologischen Expertengremium zufolge wurden diese als unwahrscheinlich im Zusammenhang mit der Impfung stehend bewertet [75]. Im Detail wurde ein Fall einer bislang unbekannten MS zugeordnet, ein Fall einer in zeitlichen Zusammenhang stehenden Meningokokkenimpfung und der dritte Fall blieb unklar [75]. Insgesamt wurden den Daten der WHO zufolge bereits 899.936.102 Impfdosen (laut WHO bis zum 22.04.2021) verabreicht [90]. An dieser Stelle muss erwähnt werden, dass im Zusammenhang mit dem AstraZeneca-Impfstoff (Vaxzervria) bis zum 04.04.2021 169 Fälle von zerebraler Venenthrombose (CVT) und 53 Fälle von splanchnischer Venenthrombose in der EU und Großbritannien gemeldet wurden (bei über 34Mio. geimpften Personen; [11, 20, 48, 64, 66, 93]). Bei den bisher dem Paul-Ehrlich-Institut (PEI) gemeldeten Fällen traten diese 
vorwiegend bei Frauen und in einem Alter $<60$ Jahren auf. Des Weiteren kam es in der Regel nach 4 bis 16 Tagen zu den Thrombosen gemeinsam mit einer Thrombozytopenie, hinweisend für eine immunologische Genese [105]. In einer Stellungnahme der Gesellschaft für Thrombose- und Hämostaseforschung (GTH) vom 01.04.2021 wurde hypothetisiert, dass es im Zuge einer impfinduzierten inflammatorischen Reaktion $\mathrm{zu}$ einer Immunstimulation mit Antikörperbildung gegen Plättchenantigene kommt. Dies führt dann, wie bei der heparininduzierten Thrombozytopenie (HIT), über den Fc-Rezeptor zu einer massiven Thrombozytenaktivierung und resultiert hier in einer prothrombotischen Thrombozytopenie [99]. Der EMA zufolge überwiegt dennoch weiterhin klar der Nutzen dem Risiko, der STIKO zufolge sollten, basierend auf den genannten Berichten, Menschen unter 60 Jahren nicht mehr mit AstraZeneca geimpft werden und jene mit bereits einer erhaltenen Dosis als 2. Dosis einen mRNA-Impfstoff erhalten [106]. Auch den Johnson\&Johnson-Impfstoff betreffend wurden 6 Fälle von CVT gemeldet. Diese stellen jedoch nach einer rezenten Überprüfung durch die FDA (Food and Drug Administration) und das CDC (Centers for Disease Control and Prevention) keinen Grund zur Revidierung der bestehenden Zulassung dar [98].

Im Allgemeinen kann aber von einem sehr guten Sicherheitsprofil der in der EU zugelassenen Impfstoffe ausgegangen werden. Dennoch können theoretisch potenzielle Langzeitnebenwirkungen der neuen genetischen Impfstoffe (Nukleinsäure- und vektorbasierte Impfstoffe) gegenwärtig noch nicht beurteilt werden, weshalb eine entsprechende strenge $\mathrm{Ob}$ servanz nach der Zulassung notwendig ist $[13,46]$.

Gegen Ende des Jahres 2020 kam jedoch im Zuge der COVID-19-Pandemie ein neues Problem auf, nämlich jenes der diversen Virusmutationen [2]. Die erste detektierte Virusvariante ist der B.1.1.7 Stamm, welcher erstmals in Großbritannien entdeckt wurde [68]. Hier wurde jedoch bis dato von keiner Resistenz gegenüber den impfstoffinduzierten neutralisierenden Antikörpern

Nervenarzt 2021 · 92:1283-1292 https://doi.org/10.1007/s00115-021-01154-5

(c) Der/die Autor(en) 2021

\section{T. Monschein · T. Zrzavy · M. Löbermann · A. Winkelmann · T. Berger · P. Rommer · H.-P. Hartung · U. K. Zettl \\ Die Corona-Pandemie und Multiple Sklerose: Impfungen und deren Implikationen für Patienten - Teil 2: Impfstofftechnologien}

\begin{abstract}
Zusammenfassung
Im Zusammenhang mit den Herausforderungen durch die weltweit vorherrschende COVID-19-Pandemie kam es zu teils epochalen Fortschritten im Bereich der Impfstofftechnologien. Neben den bereits langjährig eingesetzten Tot-, Lebend- und proteinbasierten Impfstoffen gewannen im Zuge dieser Gesundheitskrise vektorund genbasierte Impfstoffe enorm an Bedeutung. Ziel dieser Arbeit ist es daher, einen Überblick über Multiple Sklerose und Impfen, rezente Fortschritte in der SARS-
\end{abstract}

CoV-2-Impfstoff-Landschaft sowie eine detaillierte Auseinandersetzung mit den verschiedenen Impfstofftechnologien zu bieten. Abschließend sollen übersichtsmäßig klare Empfehlungen im Zusammenhang mit krankheitsmodifizierenden Therapien und Impfen bei Multiple Sklerose gegeben werden.

Schlüsselwörter

Impfstofftechnologie · Genbasiert · Vektor · COVID-19. SARS-CoV-2

\section{The corona pandemic and multiple sclerosis: vaccinations and their implications for patients-Part 2: vaccine technologies}

Abstract

Along with the challenges posed by the globally circulating COVID-19 pandemic, there have been some epochal advances in the field of vaccine technologies. In addition to the traditionally used dead, live and protein-based vaccines, vector-based and gene-based vaccines gained enormous attention in the course of this health crisis. The aim of this article is to provide an overview of multiple sclerosis (MS) and vaccination, recent advances in the SARS-CoV-2 vaccine landscape as well as a detailed discussion of the various vaccine technologies. Finally, clear recommendations in the context of diseasemodifying treatment and vaccination in MS are highlighted.

Keywords

Vaccine technologies - Gene-based - Vector . COVID-19. SARS-CoV-2 ausgegangen [18, 47]. Der B.1.351- und P.1-Stamm, die in Südafrika und Brasilien aufkamen, stellen aktuellen Expertenmeinungen zufolge wahrscheinlich kein Problem in Bezug auf die Wirksamkeit der mRNA-Impfstoffe dar [47]. Dennoch erwies sich im Labor-Setting die Variante B.1.351 als teilweise resistent gegen neutralisierende Antikörper, die durch 2 Dosen des „BNT162b2“-Impfstoffs, des „mRNA-1273“-Impfstoffs und des „NVX-CoV2373“-Impfstoffs induziert wurden [18, 68]. Der Impfstoff „ChAdOx1 nCoV-19“ scheint keine protektive Immunantwort gegen den B.1.351Stamm aufbauen zu können [41]. Ein Problem im Rahmen der Resistenzbildungen ist, dass in der Phase zwischen den ersten und zweiten Impfungen ein suboptimaler Antikörpertiter erreicht wird, welcher $\mathrm{zu}$ Resistenzbildungen prädisponiert. Demnach könnte der am 27.02.2021 von der FDA notzugelassene nur einmalig $\mathrm{zu}$ verabreichende Impfstoff "Janssen COVID-19 Vaccine“ eine attraktive Alternative/Ergänzung im $\mathrm{Zu}$ ge der Pandemiebekämpfung darstellen [97]. Erste Real-world-Daten aus Israel zeigen eine erhöhte Inzidenz an Infektionen bei zweimalig geimpften mit der Variante B.1.351 sowie bei einmalig Geimpften mit der Variante B.1.1.7. [30]. Weitere Studien sind notwendig, um die Durchbruchsinfektionen von SARSCoV-2 bei Geimpften zu verstehen [21]. Um einen möglichst effektiven und raschen Umgang mit den Virusvarianten $\mathrm{zu}$ erreichen, wurde von der $\mathrm{EH}$ eine EU-Behörde für die Krisenvorsorge und -reaktion bei gesundheitlichen Notlagen (HERA) ins Leben gerufen. Im Rahmen dessen soll auch das VACCELERATE- 


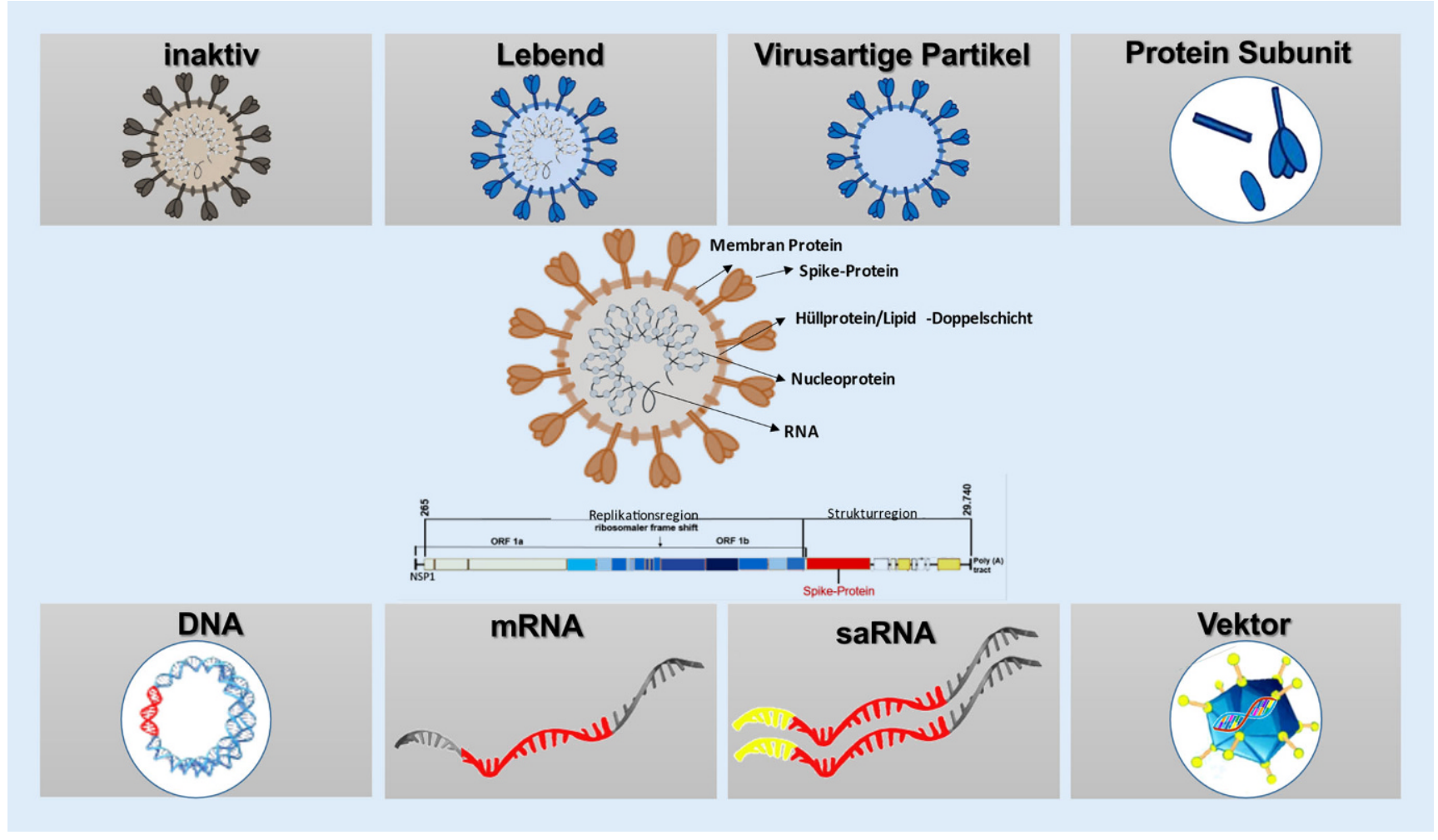

Abb. $2 \Delta$ Die aktuellen Impfstofftechnologien zur aktiven Immunisierung im Rahmen der SARS-CoV-2-Pandemie

Programm bei der Erprobung neuer/ angepasster Impfstoffen helfen [96]. Insgesamt ist jedenfalls die möglichst rasche Durchimpfung weltweit essenziell, nicht zuletzt, um die Gefahr weiterer problematischer Virusmutanten zu unterbinden.

\section{Impfstofftechnologien im Überblick}

Durch die Entwicklung und den Einsatz von Impfstoffen zur aktiven Immunisierung wurden viele Infektionskrankheiten deutlich reduziert oder nahezu vollständig verdrängt [49].

Die gegenwärtigen - zum Teil epochalen - Ansätze zur Impfstoffentwicklung beruhen auf unterschiedlichen biotechnologischen Strategien und sind insbesondere im Rahmen der SARS-CoV-2Pandemie in den Fokus eines großen öffentlichen Interesses gerückt [40, 67].

Prinzipiell unterscheiden wir in diesem Indikationsgebiet (• Abb. 2):
- inaktivierte Impfstoffe (Totimpfstoffe),

- attenuierte (abgeschwächte) Lebendimpfstoffe,

- proteinbasierte Impfstoffe,

- Virus-like-particles(VLP)-Impfstoffe,

- Subunit-(Proteinuntereinheiten-) Impfstoffe,

- Vektorimpfstoffe,

- Nukleinsäure- (genbasierte) Impfstoffe.

Toxoidimpfstoffe, wie sie zur aktiven Immunisierung gegen Tetanus sowie Diphterie eingesetzt werden, spielen im Kontext der SARS-CoV-2-Pandemie Impfstoffentwicklung keine Rolle und sollen nur aus Vollständigkeitsgründen an dieser Stelle Erwähnung finden.

\section{Inaktivierte Impfstoffe und abgeschwächte Lebendimpfstoffe}

Beide „klassischen“ Impfstoffe verwenden seit langer Zeit gut etablierte Biotechnologien und stellen für viele gegenwärtig in praxi eingesetzte Impfstoffe die Basis dar [55]. Während bei den inaktivierten Impfstoffen das genetische Material des Infektionserregers funktionsunfähig, d.h. nicht mehr replikationsfähig, ist, sind bei den attenuierten Lebendimpfstoffen die Infektionserreger zwar abgeschwächt, aber noch replikationsfähig [10, 32]. Bei beiden Impfstoffen werden nach Applikation eine Reihe von Immunprozessen initiiert, die zur Aktivierung von T-Helfer-Zellen (CD4 ${ }^{+}$T-Zellen), CD8 ${ }^{+}-\mathrm{T}-$ Zellen und B-Zellen mit konsekutiver Antikörperproduktion führen und im Endeffekt ein immunologisches Gedächtnis sowohl in T-als auch in B-Lymphozyten ( $\mathrm{T}$ - und B-MemoryZellen) zur Folge haben können [57]. Bei der Impfstoffherstellung und beim klinischen Impfstoffeinsatz sind von Erreger zu Erreger sowohl unterschiedliche „Anzüchtungs-“ und Herstellungsbedingungen als auch ein unterschiedliches Immunisierungspotenzial (Stärke und Langlebigkeit der Immunreaktion) zu bedenken. In der Regel sind Lebendimpfungen immunogener als inaktivierte Impfstoffe, sodass einerseits Zusatzfaktoren (Adjuvanzien - z. B. Aluminiumsalze) in den Impfstoff integriert werden müssen oder andererseits Booster- bzw. Auffri- 
schungsimpfungen notwendig sind, um eine klinisch relevante aktive Immunisierung zu erreichen [59]. Der Nachteil der in der Regel stärker immunogen wirkenden Lebendimpfstoffe ist, dass sie einerseits instabiler sind und andererseits bei Personen mit einem geschwächten Immunsystem gegebenenfalls zur Manifestation der Infektionskrankheit durch die abgeschwächten Impferreger führen können [57].

\section{Proteinbasierte Impfstoffe}

$\mathrm{Zu}$ dieser Impfstoffgruppe zählen Virus-like-particles(VLP)-Impfstoffe und Subunit-(Proteinuntereinheiten-)Impfstoffe. Da viele der als Antigen verwendeten hoch aufgereinigten Proteine meist nicht ausreichend immunogen wirken, müssen diesen Impfstoffen Adjuvanzien (Wirkverstärker) zugesetzt werden. So werden Komponenten des angeborenen „unspezifischen“ Immunsystems in die Nähe der eigentlichen Impfantigene gelockt und führen hier zu einer lokalen Verstärkung der Impfreaktion [57, 59]. Die Influenzavakzine sind typische proteinbasierte (Split-, Subunit-)Impfstoffe [77]. Laut WHO sind mindestens drei proteinbasierte Impfstoffe gegen SARSCoV-2 in klinischer Prüfung [108].

\section{Virus-like-particles-Impfstoffe}

Virus-like-particles(VLP)-Impfstoffe sind Pseudovirionen, die aus nichtinfektiösen Partikeln („leere“ Lipidhüllen mit Virusstrukturproteinen als Impfantigen) hergestellt werden, aber genügend virale Proteine enthalten, um eine Immunreaktion auszulösen [57].

\section{Subunit-(Proteinuntereinheiten-) Impfstoffe}

Diese Impfstoffe verwenden Teile des Infektionserregers, häufig Proteinfragmente, um die aktive Immunisierung zu initiieren. Der Vorteil ist die Minimierung von Nebenwirkungen, insbesondere des Infektionsrisikos. Nachteile sind, dass die Immunantwort in der Regel schwächer ausfällt, insbesondere, wenn molekulare Strukturen, wie „pathogen-associated molecular patterns"(PAMPs), dem Impfstoff fehlen.
Aus diesem Grund müssen diesen Impfstoffen häufig Adjuvanzien zur Verstärkung der Immunreaktion beigefügt werden. Da die geimpften Antigene keine Körperzellen infizieren, lösen diese Impfstoffe vorrangig antikörpervermittelte Immunantworten aus [57, 59].

Alle Subunit-Impfstoffe werden mithilfe moderner Biotechnologien unter Verwendung von Bakterien oder Hefen im Rahmen strenger Hygienevorschriften hergestellt. So wird für den rekombinanten Hepatitis-B-Impfstoff der genetische Code für das Impfantigen in Hefezellen integriert, durch diese in großen Mengen produziert und nach Aufreinigung als Impfantigen zur Verfügung gestellt. Durch Zusatz weiterer Komponenten, wie Konservierungsmittel und Adjuvanzien, in diesem Fall Alaun, wird der Impfstoff komplettiert $[24,74]$.

Auch Polysaccharid- oder Konjugatimpfstoffe werden mithilfe von Bakterien in Bioreaktoren produziert und anschließend durch biotechnologische Verfahren bis zur finalen Impfstoffkomponente aufbereitet [57].

\section{Vektorimpfstoffe}

Bei den Vektorimpfstoffen wird mithilfe viraler Vektoren („Taxi“, Transporter) die genetische Information für das Impfantigen in Körperzellen eingeschleust und wie bei den Nukleinsäureimpfstoffen konsekutiv von diesen gebildet. Im Genaueren führt die intramuskuläre Injektion eines rekombinanten Adenovirusimpfstoffs $\mathrm{zu}$ einer Infektion der Muskelzellen, gefolgt von der Expression des Transgens innerhalb von $24 \mathrm{~h}$, mit konkomitanter Auslösung einer angeborenen Immunantwort. Die exprimierten Proteine werden konsekutiv proteasomal abgebaut und anschließend über MHCI $\mathrm{CD}^{+}-\mathrm{T}-Z$ ellen präsentiert oder von professionellen antigenpräsentierenden Zellen (APC) aufgenommen. Schließlich wandern antigenbeladene APC zu den drainierenden Lymphknoten, wo sie in der Lage sind, $\mathrm{CD}^{+}{ }_{-}, \mathrm{CD}^{+}-\mathrm{T}$ Zellen und B-Zellen zu aktivieren, und so eine aktive Immunisierung bewirken $[14,29,39,62]$. Als virale Vektoren stehen beispielsweise das modifizier- te Vakziniavirus Ankara (MVA), das gentechnisch hergestellte vesikuläre Stomatitisvirus (rVSV) und insbesondere das Adenovirusserotyp 5 und 26 zur Verfügung.

Das Adenovirus 5 wird z. B. als Vektor für die Impfstoffe von AstraZeneca/ University of Oxford (AZD 1222) oder CanSino Biological/Beijing Institute of Biotechnology (Ad5-nCoV) für die zweizeitige Applikation verwendet. Der russische Vektorimpfstoff Sputnik-V muss ebenfalls zweimal pro Person geimpft werden. Verwendet werden hierbei aber jeweils zwei verschiedene Vektoren (Adenovirusserotyp 5 und 26), um die Immunogenität gegen den Vektor zu minimieren $[37,62,75,85]$.

Prinzipiell unterscheidet man replizierende und nichtreplizierende Vektorimpfstoffe. Nichtreplizierende Vektorimpfstoffe können keine neuen $\mathrm{Vi}$ ruspartikel (virale Vektoren) herstellen. Sie produzieren nur das Impfantigen. Im Gegensatz hierzu können replizierende Vektorimpfstoffe neue Viruspartikel in primär infizierten Körperzellen bilden, die nachfolgend weitere Zellen befallen und gemeinsam das Impfantigen bilden. Durch die hierbei induzierte Immunreaktion kommt es nachfolgend zur Limitierung der Viruspartikelreplikation [62]. Der erste in der EU zugelassene Vektorimpfstoff (November 2019) war der Ebola-Impfstoff Ervebo (rVSVZEBOV; [22]). Bisher sind ausschließlich nichtreplizierende Vektorimpfstoffe im klinischen Einsatz zur aktiven Immunisierung von Infektionserregern.

Klassisch werden virale Vektoren in Zellen gezüchtet, die an ein Substrat gebunden sind und nicht in freischwebenden Zellen. Dies ist sowohl für die Großproduktion als auch für die Skalierbarkeit von Nachteil. Aus diesem Grund geht die Forschung und Entwicklung in Richtung von Suspensionszelllinien, mit denen virale Vektoren in Bioreaktoren gezüchtet werden können. Der Zusammenbau der Vektorimpfstoffe ist ein komplexer Prozess mit Kontaminationsrisiken, die umfangreiche Sicherheitstests erforderlich machen $[19,58,61]$.

Eine besondere klinische Herausforderung der Vektorimpfstoffe stellt eine bereits im Vorfeld der Impfung erwor- 


\begin{tabular}{|c|c|c|c|c|c|c|c|}
\hline \multirow[t]{2}{*}{ DMT } & \multirow[t]{2}{*}{ Lebendimpfstoffe } & \multirow{2}{*}{$\begin{array}{l}\text { Impf- } \\
\text { zeitpunkt }\end{array}$} & \multirow[t]{2}{*}{ Totimpfstoffe } & \multicolumn{2}{|c|}{$\begin{array}{l}\text { Gen - } \\
\text { basiert [101] }\end{array}$} & \multirow{2}{*}{$\begin{array}{l}\text { Impf- } \\
\text { zeitpunkt }\end{array}$} & \multirow[t]{2}{*}{ Immunantwort } \\
\hline & & & & mRNA & Vektor & & \\
\hline HDMP' & kontraindiziert [100] & $\begin{array}{l}\geq 1 \\
\text { Monat [79] }\end{array}$ & $J A$ & $J A$ & $J A^{\prime \prime}$ & $\begin{array}{l}\text { Therapie } \\
\text { Ende }\end{array}$ & $\begin{array}{l}\text { möglicherweise } \\
\text { reduziert[50] }\end{array}$ \\
\hline ß-Interferone & \begin{tabular}{|l} 
strikte \\
Indikation [100] \\
\end{tabular} & $\begin{array}{l}\geq 1 \\
\text { Monat [79] }\end{array}$ & $J A$ & JA & $J A^{\prime \prime}$ & jederzeit & ähnlich [65] \\
\hline Glatirameracetat & \begin{tabular}{|l} 
strikte \\
Indikation [100]
\end{tabular} & $\begin{array}{l}\text { keine } \\
\text { Angabe }\end{array}$ & $J A$ & $J A$ & $J A^{\prime \prime}$ & jederzeit & ähnlich [53] \\
\hline Dimethylfumarat & $\begin{array}{l}\text { strikte } \\
\text { Indikation [100] }\end{array}$ & $\begin{array}{l}\geq 3 \\
\text { Monate[79] }\end{array}$ & $J A$ & $J A$ & $J A^{\prime \prime}$ & jederzeit & ähnlich [73] \\
\hline Teriflunomid & kontraindiziert [100] & IV 2 Jahre $[79]$ & $J A$ & $J A$ & $J A^{\prime \prime}$ & jederzeit & $\begin{array}{l}\text { leicht } \\
\text { reduziert }[4,5]\end{array}$ \\
\hline $\begin{array}{l}\text { S1P- } \\
\text { Modulatoren }^{a}\end{array}$ & kontraindiziert [100] & $\begin{array}{l}\geq 3 \\
\text { Monate [79] }\end{array}$ & $J A$ & $\mathrm{JA}$ & $J A^{\prime \prime}$ & jederzeit & $\begin{array}{l}\text { reduziert [27, } \\
44]\end{array}$ \\
\hline Natalizumab & kontraindiziert [100] & $\begin{array}{l}\geq 3 \\
\text { Monate[79] }\end{array}$ & $J A$ & $J A$ & $J A^{\prime \prime}$ & jederzeit & ähnlich $[28,72]$ \\
\hline $\begin{array}{l}\text { B-Zell- } \\
\text { depletierende } \\
\text { Therapien }^{\text {b }}\end{array}$ & kontraindiziert [100] & $\begin{array}{l}\geq 12 \\
\text { Monate [79] }\end{array}$ & $J A$ & $J A$ & $J A^{\prime \prime}$ & $\begin{array}{l}\geq 4-6 \\
\text { Monate[100] }\end{array}$ & reduziert $[6,7]$ \\
\hline Alemtuzumab & kontraindiziert [100] & $\begin{array}{l}\geq 12 \\
\text { Monate [79] }\end{array}$ & $J A$ & $J A$ & $J A^{\prime \prime}$ & $\begin{array}{l}\text { keine } \\
\text { Angabev }\end{array}$ & reduziert[44] \\
\hline Cladribin & kontraindiziert [100] & spezifiziert $^{\mathrm{VI}}$ & $J A$ & $J A$ & $J A^{\prime \prime}$ & $\begin{array}{l}\text { keine } \\
\text { Angabev }^{\text {v }}\end{array}$ & $\begin{array}{l}\text { möglicherweise } \\
\text { reduziert }[1,100]\end{array}$ \\
\hline Mitoxantron & kontraindiziert [100] & $\begin{array}{l}\geq 3 \\
\text { Monate[100] }\end{array}$ & $J A$ & $\mathrm{JA}$ & $J A^{\prime \prime}$ & $\begin{array}{l}\geq 3 \\
\text { Monate[100] }\end{array}$ & $\begin{array}{l}\text { möglicherweise } \\
\text { reduziert[100] }\end{array}$ \\
\hline $\begin{array}{l}\text { I HDMP = hochdosi } \\
\text { II nicht-replizieren } \\
\text { III nach Therapieen } \\
\text { IV Auswaschmögl } \\
\text { anschließende Kont } \\
\text { von 11⁄2 Monaten zw } \\
\text { V } \geq 3-6 \text { Monate jen } \\
\text { VI bis die Anzahl de } \\
\text { a Sphingosin-1-Pho } \\
\text { nicht zugelassen) } \\
\text { b inkludierend Ritux }\end{array}$ & $\begin{array}{l}\text { tes Methylprednisolon } \\
\text { e Vektor-Impfstoffe } \\
\text { te oder nach Dosisredukt } \\
\text { hkeit mit Aktivkohle ode } \\
\text { lle des Plasmaspiegels d } \\
\text { schen der ersten Messuns } \\
\text { ach regionalen Empfehlu } \\
\text { Leukozyten/Lymphozyte } \\
\text { phat-Rezeptor-Modulatc } \\
\text { nab, Ocrelizumab und O }\end{array}$ & $\begin{array}{l}\text { ion auf Predniso } \\
\text { r Cholestyramin, } \\
\text { urch zwei getren } \\
\text { geines Plasmasp } \\
\text { ngen [104, 102, } \\
\text { innerhalb norn } \\
\text { ren: Fingolimod, } \\
\text { fatumumab }\end{array}$ & $\begin{array}{l}\text { äquivalent }<20 \mathrm{~m} \\
\text { Inabhängig vom } \\
\text { te Tests im Abstan } \\
\text { gels unter } 0,02 \mathrm{~m} \\
\text { 03] } \\
\text { aler Grenzen liegt } \\
\text { Siponimod, Ozani }\end{array}$ & $\begin{array}{l}\text { /Tag bei } \\
\text { ewählten } \\
d \text { von min } \\
\text { /l und de } \\
{[92]} \\
\text { nod und } F\end{array}$ & $\begin{array}{l}\text { rwachsen } \\
\text { Auswasch } \\
\text { lestens } 14 \\
\text { Lebendim }\end{array}$ & $\begin{array}{l}\text { [79] } \\
\text { agfahren ist eine } \\
\text { fung erforderlich }\end{array}$ & $\begin{array}{l}\text { artezeit } \\
{[79]}\end{array}$ \\
\hline
\end{tabular}

Abb. 3 ॥ Impfempfehlungen für Lebend-/Tot-und genbasierte Impfstoffe in Bezug aufdie jeweilige Immuntherapiemit dem jeweiligen Verabreichungszeitpunkt sowie zu erwartender Immunantwort

ben Immunantwort gegen den viralen Vektor dar, die zu einer verringerten aktiven Immunisierung (Impfantwort) gegen das Impfantigen führen kann [17]. Diese potenzielle Antivektorimmunität macht es zudem problematisch, eine notwendige Booster-Immunisierung (zweite oder mehrfache Impfung) mit dem gleichen Vektor durchzuführen. Dies könnte ein möglicher Grund für die prozentual niedrigere Wirksamkeit des AstraZeneca-Impfstoffs sein. Die präexistierende oder durch die aktive Erstimmunisierung induzierte spezifische Immunantwort gegen den viralen Impfvektor stellt jedenfalls eine besondere Herausforderung im klinischen Großeinsatz dar und ist aktuell noch nicht abschließend zu bewerten.

\section{Genbasierte Impfstoffe}

Genbasierte Impfstoffe, auch Nukleinsäureimpfstoffe genannt, verwenden entweder mRNA (Messenger-Ribonukleinsäure) oder DNA (Desoxyribonukleinsäure), um Körperzellen die Anleitung zur Produktion des Impfantigens zu geben und nachfolgend die aktive Immunisierung zu initiieren $[26,69]$. Das heißt, 
Tab. 1 Überblick über die jeweiligen Fachgesellschaften der jeweiligen Länder im Deutsch-sprachigen Raum mit den entsprechenden Links

\section{Land - Fachgesellschaft Weiterführende Links}

Österreich - ÖGN, ÖMSG https://www.oegn.at/covid-19/covid19-und-multiple-sklerose-ms/ https://www.oemsg.at/news/

Deutschland-DGN, KKNMS https://dgn.org/ https://www.kompetenznetz-multiplesklerose.de/

Schweiz - SNG, SMSG https://www.multiplesklerose.ch/de/aktuelles/detail/ informationssammlung-zu-ms-corona/

der Körper der geimpften Person stellt, wie bei den Vektorimpfstoffen, selbst das Impfantigen her [57]. Bei DNA-Impfstoffen wird die das Antigen kodierende DNA chemisch synthetisiert und mithilfe spezifischer Enzyme in ein Bakterienplasmid inseriert. Das rasante Kopieren dieses Plasmids erfolgt initial in sich schnell teilenden Bakterien, mit nachfolgender Plasmidisolierung und -aufreinigung [33, 83]. Bei mRNA-Impfstoffen wird in der Regel die genetische Information (mRNA) für das Impfantigen (Protein) in das Zytosol von Körperzellen eingeschleust [54].

Um den Eintritt ins Zytosol zu erleichtern, kann die relativ instabile mRNA in Liposomen oder Lipidnanopartikel (LNP) verpackt werden. Im Zytosol wird die mRNA an Ribosomen gebunden und die Bildung eines Polypeptids (Impfantigen) katalysiert. Das Impfantigen wird von den produzierenden Körperzellen an der Zelloberfläche dargeboten und/ oder von diesen Körperzellen liberiert. Über beide Wege wird das Impfantigen von Immunzellen erkannt. Ziel der immunologischen Prozessierung ist die Generierung von T- und B-Gedächtnislymphozyten („memory cells“; [43, 54]). Eine Modifikation von mRNAImpfstoffen besteht darin, dass als genetischer Bauplan für das Impfantigen selbstreplizierende oder selbstamplifizierende RNA (saRNA) verwendet wird. Dies ist beispielsweise bei dem Impfstoff „BNT 162c2“ der Fall (im Gegensatz $\mathrm{zu}$ „BNT162b2“ noch nicht zugelassen; $[8,60])$. saRNA-Impfstoffe stammen vom alphaviralen Genom mit zwei offenen Leserahmen („open reading frame“ [ORF]; [9]). Der erste ORF codiert für die RNA-abhängige RNA-Polymerase (Replikase) und der zweite ORF für das Strukturprotein, in diesem Fall das
Impfantigen, z. B. das Spike-Protein des SARS-CoV-2.

Der Vorteil dieser nukleinsäurebasierten Technologie ist die relativ einfache und schnelle Herstellung der genbasierten Impfstoffe, sobald die Nukleinsäuresequenz des Impfantigens bekannt ist. Dies ist insbesondere von großer praktischer Relevanz, wenn neu auftretende epidemisch, pandemisch oder schnell mutierende Infektionserreger eine Rolle spielen [54]. Nachteile der mRNA-Impfstoffe sind einerseits logistische Probleme (Lagerung und Transport bei -20 bis unter $-70^{\circ} \mathrm{C}$ ) und andererseits die sehr begrenzten praktischen Erfahrungen bzw. fehlenden Langzeitergebnisse mit diesem Impfstofftyp im Rahmen von Infektionserkrankungen [54].

Im Rahmen der SARS-CoV-2-Pandemie sind in der EU bisher 2 humane mRNA-Impfstoffe (von BioNTech/Pfizer [BNT 162b2; Tozinameran, Comirnaty] am 21.12.2020 und von Moderna Biotech [mRNA-1273; Moderna COVID-19 Vaccine] am 06.01.2021) zugelassen worden $[3,56]$. Bisher gibt es keine Zulassung für einen humanen DNA-Impfstoff gegen eine Infektionserkrankung.

\section{Immuntherapien (DMTs) in der MS und Impfen}

Allgemein sollten stets vor der Initiierung einer DMT der aktuelle Immunstatus genauso wie mögliche Kontraindikationen, insbesondere Lebendimpfstoffe betreffend, berücksichtigt werden $[34,36]$. Für Totimpfstoffe wird ein Mindestabstand von 2 Wochen und für Lebendimpfstoffe von 4 Wochen vor Initiierung einer Immuntherapie empfohlen. Um die Wahrscheinlichkeit einer möglichst potenten Immunantwort $\mathrm{zu}$ erhöhen sind jedoch unabhängig vom Impfstoff 6 Wochen zu bevorzugen, sofern dies die individuelle Situation erlaubt [63, 104]. Gerade Menschen mit Multipler Sklerose werden alle Impfungen entsprechend den regionalen Empfehlungen empfohlen, um so die Infektionswahrscheinlichkeit und die damit verbundenen Risiken zu reduzieren [16, 31, 107]. Eine Ausnahme stellt die Gelbfieberimpfung dar, da hier die Auslösung eines Schubes durch die Impfung nicht ausgeschlossen werden kann [15]. Eine Absprache mit dem behandelnden Neurologen sowie entsprechende Risiko-Nutzen-Evaluation werden empfohlen.

Einen Überblick über die Empfehlungen für Lebend-/Tot- und genbasierte Impfstoffe in Abhängigkeit von der jeweiligen DMT gibt • Abb. 3. Des Weiteren ist es wichtig, die entsprechenden Zeitintervalle einzuhalten, um bei Lebendimpfstoffen eine potenzielle Erkrankung zu verhindern und bei andersartigen Impfstoffen eine protektive Immunantwort aufbauen zu können. Insbesondere bei B-Zell-depletierenden Therapien ist bei T-Zell-unabhängigen Antigenen von einer reduzierten Immunantwort auszugehen (VELOCE-Studie; [6]).

Bezugnehmend auf die SARS-CoV-2Impfstoffe gilt es zu erwähnen, dass in sämtlichen Zulassungsstudien Patienten mit Autoimmunerkrankungen unter Immuntherapie nicht berücksichtigt wurden und dies somit einen klaren Limitierungsfaktor darstellt.

\section{Schlussfolgerungen}

Im Zuge der rezenten COVID-19-Pandemie wurden bisher 2 mRNA-Impfstoffe sowie 2 nichtreplizierende Vektorvirusimpfstoffe von der Europäischen Arzneimittelbehörde (EMA) zugelassen [3, $56,75,94]$. Diese zeigten allesamt eine sehr gute Wirksamkeit sowie auch ein sehr gutes Sicherheitsprofil. Somit kann anschließend an die Empfehlungen der Internationalen Multiple Sklerose Vereinigung (MSIF) eine klare Empfehlung zur Impfung für alle MS-Patienten, immer in Rücksprache mit dem behandelnden Neurologen, ausgesprochen werden [101]. Die rasche Durchimpfung der Bevölkerung, insbesondere Menschen mit MS, zählt zu den wichtigsten Maßnah- 
men im Kampf gegen die dominierende COVID-19-Pandemie (• Tab. 1).

\section{Korrespondenzadresse}

\section{Tobias Monschein}

Universitätsklinik für Neurologie, Medizinische

Universität Wien

Waehringer Guertel 18-20, 1090 Wien,

Österreich

tobias.monschein@meduniwien.ac.at

\section{Prof. Hans-Peter Hartung, FRCP}

Klinik für Neurologie, Universitätsklinikum

Düsseldorf, Medizinische Fakultät, Heinrich-

Heine-Universität

Moorenstraße 5, 40225 Düsseldorf,

Deutschland

hans-peter.hartung@uni-duesseldorf.de

Funding. Open access funding provided by Medical University of Vienna.

\section{Einhaltung ethischer Richtlinien}

Interessenkonflikt. T. Monschein, T. Zrzavy, M. Löbermann, A. Winkelmann, T. Berger, P. Rommer, H.P. Hartung und U.K. Zettl geben an, dass kein Interessenkonflikt besteht.

Für diesen Beitrag wurden von den Autoren keine Studien an Menschen oder Tieren durchgeführt. Für die aufgeführten Studien gelten die jeweils dort angegebenen ethischen Richtlinien.

Open Access. Dieser Artikel wird unter der Creative Commons Namensnennung 4.0 International Lizenz veröffentlicht, welche die Nutzung, Vervielfältigung, Bearbeitung, Verbreitung und Wiedergabe in jeglichem Medium und Format erlaubt, sofern Sie den/die ursprünglichen Autor(en) und die Quelle ordnungsgemäß nennen, einen Link zur Creative Commons Lizenz beifügen und angeben, ob Änderungen vorgenommen wurden.

Die in diesem Artikel enthaltenen Bilder und sonstiges Drittmaterial unterliegen ebenfalls der genannten Creative Commons Lizenz, sofern sich aus der Abbildungslegende nichts anderes ergibt. Sofern das betreffende Material nicht unter der genannten Creative Commons Lizenz steht und die betreffende Handlung nicht nach gesetzlichen Vorschriften erlaubt ist, ist für die oben aufgeführten Weiterverwendungen des $\mathrm{Ma}$ terials die Einwilligung des jeweiligen Rechteinhabers einzuholen.

Weitere Details zur Lizenz entnehmen Sie bitte der Lizenzinformation auf http://creativecommons.org/ licenses/by/4.0/deed.de.

\section{Literatur}

1. Achiron A, Mandel M, Dreyer-alster S et al (2021) Humoral immune response to COVID-19 mRNA vaccine in patients with multiple sclerosis treated with high-efficacy disease-modifying therapies.
Ther Adv Neurol Disord 2021;14:1-8. https://doi. org/10.1177/17562864211012835

2. Altmann DM, Boyton RJ, Beale R (2021) Immunity to SARS-CoV-2 variants of concern. Science 371(80):1103-1104. https://doi.org/10.1126/ science.abg7404

3. Baden LR, El Sahly HM, Essink B et al (2021) Efficacy and Safety of the mRNA-1273 SARS-CoV-2 Vaccine. N Engl J Med 384:403-416. https://doi.org/10. 1056/nejmoa2035389

4. Bar-Or A, Freedman MS, Kremenchutzky M et al (2013) Teriflunomide effect on immune response to influenza vaccine in patients with multiple sclerosis. Neurology 81:552-558. https://doi.org/ 10.1212/WNL.0b013e31829e6fbf

5. Bar-OrA, WiendlH,MillerBetal (2015) Randomized study of teriflunomide effects on immune responses to neoantigen and recall antigens. Neurol Neuroimmunol Neuroinflamm 2:e70. https://doi.org/10.1212/NXI.0000000000000070

6. Bar-Or A, Calkwood JC, Chognot C et al (2020) Effect of ocrelizumab on vaccine responses in patients with multiple sclerosis: the VELOCE study. Neurology 95:e1999-e2008. https://doi.org/10. 1212/WNL.0000000000010380

7. Bingham CO, Looney RJ, Deodhar A et al (2010) Immunization responses in rheumatoid arthritis patients treated with rituximab: results from a controlled clinical trial. Arthritis Rheum 62:64-74. https://doi.org/10.1002/art.25034

8. Blakney AK, McKay PF (2021) Next-generation COVID-19 vaccines: here come the proteins. Lancet 397:643-645. https://doi.org/10.1016/S01406736(21)00258-0

9. Bloom K, van den Berg F, Arbuthnot P (2020) Selfamplifying RNA vaccines for infectious diseases. Gene Ther. https://doi.org/10.1038/s41434-02000204-y

10. Broadbent AJ, Santos CP, Anafu A et al (2016) Evaluation of the attenuation, immunogenicity, and efficacy of a live virus vaccine generated by codon-pair bias de-optimization of the 2009 pandemic $\mathrm{H} 1 \mathrm{~N} 1$ influenza virus, in ferrets. Vaccine 34:563-570. https://doi.org/10.1016/j.vaccine. 2015.11.054

11. Cines DB, Bussel JB (2021) E d i t o r i a I SARS-CoV-2 vaccine-induced immune thrombotic thrombocytopenia https://doi.org/10.1056/NEJM

12. Confavreux C, Suissa S, Saddier P et al (2001) Vaccinations and the risk of relapse in multiple sclerosis. N Engl J Med 344:319-326. https://doi. org/10.1056/nejm200102013440501

13. van Doremalen $N$, Lambe $T$, Spencer $A$ et al (2020) ChAdOx1 nCoV-19 vaccine prevents SARSCoV-2 pneumonia in rhesus macaques. Nature 586:578-582. https://doi.org/10.1038/s41586020-2608-y

14. Ewer KJ, LambeT, Rollier CSet al (2016) Viralvectors as vaccine platforms: From immunogenicity to impact. Curr Opin Immunol 41:47-54. https://doi. org/10.1016/j.coi.2016.05.014

15. Farez MF, Correale J (2011) Yellow fever vaccination and increased relapse rate in travelers with multiple sclerosis. Arch Neurol 68:1267-1271. https://doi.org/10.1001/archneurol.2011.131

16. Farez MF, Correale J, Armstrong MJ et al (2019) Practice guideline update summary: vaccine-preventable infections and immunization in multiple sclerosis: report of the guideline development, dissemination, and implementation subcommittee of the American academy of neurology. Neurology 93:584-594. https://doi.org/10.1212/ WNL.0000000000008157
17. Fausther-Bovendo H, Kobinger GP (2014) Preexisting immunity against Ad vectors: Humoral, cellular, and innate response, what's important? Hum Vaccines Immunother 10:2875-2884.https:// doi.org/10.4161/hv.29594

18. Garcia-Beltran WF, Lam EC, Denis SK et al (2021) Multiple SARS-CoV-2 variants escape neutralization by vaccine-induced humoral immunity. Cell. https://doi.org/10.1016/j.cell. 2021.03.013

19. Genzel Y (2015) Designing cell lines for viral vaccine production: where do we stand? Biotechnol J 10:728-740. https://doi.org/10.1002/biot. 201400388

20. Greinacher A, Thiele T, Warkentin TE, Weisser K (2021) A prothrombotic Thrombocytopenic disorder resembling heparin-induced Thrombocytopenia following Coronavirus-19 vaccination. Res Sq Preprint 1-8. https://doi.org/10.21203/rs.3.rs362354/v1

21. Hacisuleyman E, Hale C, Saito $Y$ et al (2021) Vaccine breakthrough infections with SARS-coV-2 variants. N Engl J Med. https://doi.org/10.1056/ NEJMoa2105000

22. Halperin SA, Das R, Onorato MT et al (2019) Immunogenicity, lot consistency, and extended safety of rVSVDG-ZEBOV-GP vaccine: a phase 3 randomized, double-blind, placebo-controlled study in healthy adults. JInfect Dis 220:1127-1135. https://doi.org/10.1093/infdis/jiz241

23. Hapfelmeier A, Gasperi C, Donnachie E, Hemmer B (2019) A large case-control study on vaccination as risk factor for multiple sclerosis. Neurology 93:e908-e916. https://doi.org/10.1212/WNL. 0000000000008012

24. Hartmann K, Keller-Stanislawski B (2002) Rekombinante Hepatitis-B-Impfstoffe und Verdachtsfälle unerwünschter Reaktionen Eine Bewertung der Spontanerfassungsdaten des Paul-EhrlichInstituts 1995 bis 2000: Eine Bewertung der Spontanerfassungsdaten des Paul-Ehrlich-Instituts 1995 bis 2000. Bundesgesundheitsblatt Gesundheitsforschung Gesundheitsschutz 45:355-363. https://doi.org/10.1007/s00103-002-0388-1

25. Hernán MA, Jick SS, Olek MJ, Jick H (2004) Recombinant hepatitis $B$ vaccine and the risk of multiple sclerosis: A prospective study. Neurology 63:838-842. https://doi.org/10.1212/01.WNL. 0000138433.61870 .82

26. lavarone C, O'hagan DT, Yu D et al (2017) Mechanism of action of mRNA-based vaccines. Expert Rev Vaccines 16:871-881. https://doi.org/ 10.1080/14760584.2017.1355245

27. Kappos L, Mehling M, Arroyo R et al (2015) Randomized trial of vaccination in fingolimodtreated patients with multiple sclerosis. Neurology 84:872-879. https://doi.org/10.1212/WNL. 0000000000001302

28. Kaufman M, Pardo G, Rossman H et al (2014) Natalizumab treatment shows no clinically meaningful effects on immunization responses in patients with relapsing-remitting multiple sclerosis. J Neurol Sci 341:22-27. https://doi.org/ 10.1016/j.jns.2014.03.035

29. Kim SH, Samal SK (2016) Newcastle disease virus as a vaccine vector for development of human and veterinary vaccines. Viruses. https://doi.org/10. 3390/v8070183

30. Kustin T, Harel N, Finkel U et al (2021) Evidence for increased breakthrough rates of SARS-CoV-2 variants of concern in BNT162b2 mRNA vaccinated individuals. medRxiv:2021.04.06.21254882

31. Lebrun C, Vukusic S (2019) Immunization and multiple sclerosis: recommendations from the 
French multiple sclerosis society. Mult Scler Relat Disord 31:173-188. https://doi.org/10.1016/j. msard.2019.04.004

32. Lee N-H, Lee J-A, Park S-Y et al (2012) A review of vaccine development and research for industry animals in Korea. Clin Exp Vaccine Res 1:18. https:// doi.org/10.7774/cevr.2012.1.1.18

33. Lee YLY, Izzard L, Hurt AC (2018) A review of DNA vaccines againstinfluenza. Front Immunol.https:// doi.org/10.3389/fimmu.2018.01568

34. Löbermann M, Boršo D, Hilgendorf I et al (2012) Immunization in the adult immunocompromised host. Autoimmun Rev 11:212-218. https://doi. org/10.1016/j.autrev.2011.05.015

35. Löbermann M, Handorn B, Winkelmann A et al (2018) Multiple sclerosis and hepatitis B vaccination: what does the verdict of the European court of justice on liability after vaccination mean? Nervenarzt 89:1172-1178. https://doi.org/10. 1007/s00115-018-0492-6

36. Loebermann M, Winkelmann A, Hartung HP et al (2012) Vaccination against infection in patients with multiple sclerosis. Nat Rev Neurol 8:143-151. https://doi.org/10.1038/nrneurol.2012.8

37. Logunov DY, Dolzhikova IV, Shcheblyakov DV et al (2021) Safety and efficacy of an rAd26 and rAd5 vector-based heterologous primeboost COVID-19 vaccine: an interim analysis of a randomised controlled phase 3 trial in Russia. Lancet 397:671-681. https://doi.org/10.1016/ S0140-6736(21)00234-8

38. Logunov DY, Dolzhikova IV, Zubkova OV et al (2020) Safety and immunogenicity of an rAd26 and $\mathrm{rAd} 5$ vector-based heterologous prime-boost COVID-19 vaccine in two formulations: two open, non-randomised phase 1/2 studies from Russia. Lancet 396:887-897. https://doi.org/10.1016/ S0140-6736(20)31866-3

39. Lundstrom K (2021) Viral vectors for COVID-19 vaccine development. Viruses. https://doi.org/10. 3390/v13020317

40. Lurie N, Saville M, Hatchett R, Halton J (2020) Developing Covid-19 vaccines at pandemic speed. N Engl J Med 382:1969-1973. https://doi.org/10. 1056/nejmp2005630

41. Madhi SA, Baillie V, Cutland CL et al (2021) Efficacy of the ChAdOx1 nCoV-19 Covid-19 vaccine against the B.1.351 variant. N Engl J Med. https://doi.org/ 10.1056/nejmoa2102214

42. Mailand MT, Frederiksen JL (2017) Vaccines and multiple sclerosis: a systematic review. J Neurol 264:1035-1050. https://doi.org/10.1007/s00415016-8263-4

43. Maruggi G, Zhang C, Li J et al (2019) mRNA as a transformative technology for vaccine development to control infectious diseases. Mol Ther 27:757-772. https://doi.org/10.1016/j. ymthe.2019.01.020

44. McCarthy CL, Tuohy $\mathrm{O}$, Compston DAS et al (2013) Immune competence after alemtuzumab treatment of multiple sclerosis. Neurology $81: 872-876$. https://doi.org/10.1212/WNL. 0b013e3182a35215

45. Mehling M, Hilbert P, Fritz $S$ et al (2011) Antigen-specific adaptive immune responses in fingolimod-treated multiple sclerosis patients. Ann Neurol 69:408-413. https://doi.org/10.1002/ ana.22352

46. Mitani K, Kubo S (2006) Adenovirus as an integrating vector. Curr Gene Ther 2:135-144. https://doi.org/10.2174/1566523024605591

47. Moore JP (2021) Approaches for optimal use of different COVID-19 vaccines: issues of viral variants and vaccine efficacy. JAMA 325:1251-1252 https://doi.org/10.1001/jama.2021.3465

48. MuirK-L, KallamA, KoepsellSA, GundaboluK(2021) Thrombotic Thrombocytopenia after Ad26.COV2.S vaccination. N Engl J Med 384(20):1964-1965. https://doi.org/10.1056/NEJMc2105869

49. Nabel GJ (2013) Designing tomorrow's vaccines. N Engl J Med 368:551-560. https://doi.org/10. 1056/nejmra1204186

50. National Center for Immunization (2011) General recommendations on immunization-recommendations of the Advisory Committee on Immunization Practices (ACIP). MMWR Recomm Rep 60:1-64

51. NCT04471519 (2020) Whole-Virion Inactivated SARS-CoV-2 Vaccine (BBV152) for COVID-19 in Healthy Volunteers. https://clinicaltrials.gov/ show/NCT04471519

52. NCT04527575 (2020) Study of the Safety, Reactogenicity and Immunogenicity of "EpiVacCorona” Vaccine for the Prevention of COVID-19. https:// clinicaltrials.gov/show/NCT04527575

53. Olberg HK, Eide GE, Cox RJ et al (2018) Antibody response to seasonal influenza vaccination in patients with multiple sclerosis receiving immunomodulatory therapy. Eur J Neurol 25:527-534. https://doi.org/10.1111/ene.13537

54. Pardi N, Hogan MJ, Porter FW, Weissman D (2018) mRNA vaccines-a new era in vaccinology. Nat Rev Drug Discov 17:261-279. https://doi.org/10.1038/ nrd.2017.243

55. Plotkin S, Orenstein W, Offit P, Kathryn ME (2018) Plotkin's vaccines, 7. Aufl.

56. Polack FP, Thomas SJ, Kitchin N et al (2020) Safety and efficacy of the BNT162b2 mRNA Covid-19 vaccine. N Engl J Med 383:2603-2615. https://doi. org/10.1056/nejmoa2034577

57. Pollard AJ, Bijker EM (2021) A guide to vaccinology: from basic principles to new developments. Nat Rev Immunol 21:83-100. https://doi.org/10.1038/ s41577-020-00479-7

58. Rauch S, Jasny E, Schmidt KE, Petsch B (2018) New vaccine technologies to combat outbreak situations. Front Immunol. https://doi.org/10. 3389/fimmu.2018.01963

59. Reed SG, Orr MT, Fox CB (2013) Key roles of adjuvants in modern vaccines. Nat Med 19:1597-1608. https://doi.org/10.1038/nm.3409

60. Rego GNA, Nucci MP, Alves AH et al (2020) Current clinical trials protocols and the global effort for immunization against sars-cov-2. Vaccines 8:1-44. https://doi.org/10.3390/vaccines8030474

61. Rodrigues AF, Soares HR, Guerreiro MR et al (2015) Viral vaccines and their manufacturing cell substrates: New trends and designs in modern vaccinology. Biotechnol J 10:1329-1344. https:// doi.org/10.1002/biot.201400387

62. Rollier CS, Reyes-Sandoval A, Cottingham MG et al (2011) Viral vectors as vaccine platforms: Deployment in sight. Curr Opin Immunol 23:377-382. https://doi.org/10.1016/j.coi.2011.03.006

63. Rubin LG, Levin MJ, Ljungman P et al (2014) Executive summary:2013IDSA clinical practice guideline for vaccination of the Immunocompromised host. Clin Infect Dis 58:309-318. https://doi.org/10. 1093/cid/cit816

64. Schultz NH, Sørvoll IH, Michelsen AE et al (2021) Thrombosis and thrombocytopenia after ChAdox 1 ncoV-19 vaccination. N Engl J Med. https://doi.org/ 10.1056/nejmoa2104882

65. Schwid SR, Decker MD, Lopez-Bresnahan M (2005) Immune response to influenza vaccine is maintained in patients with multiple sclerosis receiving interferon beta-1a. Neurology
65:1964-1966. https://doi.org/10.1212/01.wnl. 0000188901.12700.e0

66. Scully M, Singh D, Lown R et al (2021) Pathologic antibodies to platelet factor 4 after ChAdox 1 ncoV19 vaccination. N Engl J Med. https://doi.org/10. 1056/NEJMoa2105385

67. Sharma O, Sultan AA, Ding H, Triggle CR (2020) $A$ review of the progress and challenges of developing a vaccineforCOVID-19. Front Immunol. https://doi.org/10.3389/fimmu.2020.585354

68. Shen X, Tang H, McDanal C et al (2021) SARSCoV-2 variant B.1.1.7 is susceptible to neutralizing antibodies elicited by ancestral spike vaccines. SSRN Electron J. https://doi.org/10.2139/ssrn. 3777473

69. Silveira MA, Tav C, Bérube-Simard FA et al (2021) Modulating HSF1 levels impacts expression of the estrogen receptor $a$ and antiestrogen response. Life Sci Alliance. https://doi.org/10.26508/lsa. 202000811

70. Taylor B, Miller E, Farrington CP et al (1999) Autism and measles, mumps, and rubella vaccine: no epidemiological evidence for a causal association. Lancet 353:2026-2029. https://doi.org/10.1016/ S0140-6736(99)01239-8

71. Taylor LE, Swerdfeger AL, Eslick GD (2014) Vaccines are not associated with autism: an evidence-based meta-analysis of case-control and cohort studies. Vaccine 32:3623-3629. https://doi.org/10.1016/j. vaccine.2014.04.085

72. Vågberg M, Kumlin U, Svenningsson A (2012) Humoral immune response to influenza vaccine in natalizumab-treated MS patients. Neurol Res 34:730-733. https://doi.org/10.1179/ 1743132812Y.0000000059

73. Von Hehn C, Howard J, Liu S et al (2018) Immune response to vaccines is maintained in patients treated with dimethyl fumarate. Neuro Neuroimmunol Neuroinflamm. https://doi.org/ 10.1212/NXI.0000000000000409

74. Vartak A, Sucheck SJ (2016) Recent advances in subunit vaccine carriers. Vaccines. https://doi.org/ 10.3390/vaccines4020012

75. Voysey M, Clemens SAC, Madhi SA et al (2021) Safety and efficacy of the ChAdOx1 nCoV-19 vaccine (AZD1222) against SARS-CoV-2: an interim analysis of four randomised controlled trials in Brazil, South Africa, and the UK. Lancet397:99-111. https://doi.org/10.1016/S0140-6736(20)32661-1

76. Wakefield AJ, Murch SH, Anthony A et al (1998) Retracted: Ileal-lymphoid-nodular hyperplasia, non-specific colitis, and pervasive developmental disorder in children. Lancet 351:637-641. https:// doi.org/10.1016/S0140-6736(97)11096-0

77. Wei CJ, Crank MC, Shiver J et al (2020) Nextgeneration influenza vaccines: opportunities and challenges. Nat Rev Drug Discov 19:239-252. https://doi.org/10.1038/s41573-019-0056-x

78. Wiedermann U, Kollaritsch $\mathrm{H}$, Bachinger $\mathrm{G}$ et al (2014) Reaktionen und Nebenwirkungen nach Impfungen: Erläuterungen und Definitionen in Ergänzung zum Österreichischen Impfplan. Intrinsic Act 2:e2. https://doi.org/10.25006/ia.2.1e2

79. Wiedermann $\mathrm{U}$, Sitte $\mathrm{HH}$, Burgmann $\mathrm{H}$ et al (2016) Guidelines for vaccination of immunocompromised individuals. Wien Klin Wochenschr 128:337-376. https://doi.org/10.1007/s00508016-1033-6

80. Wiedermann-Schmidt U (2016) Impfen Wann. Wogegen. Warum

81. Xia S, Zhang Y, Wang Y et al (2021) Safety and immunogenicity of an inactivated SARS-CoV-2 vaccine, BBIBP-CorV: a randomised, double-blind, 
placebo-controlled, phase 1/2 trial. Lancet Infect Dis 21:39-51. https://doi.org/10.1016/S14733099(20)30831-8

82. Yang S, Li Y, Dai L et al (2020) Safety and immunogenicity of a recombinant tandem-repeat dimeric RBD protein vaccine against COVID-19 in adults: Pooled analysis of two randomized, double-blind, placebo-controlled, phase 1 and 2 trials. medRxiv. https://doi.org/10.1101/2020.12. 20.20248602

83. Yurina V(2018) Live bacterialvectors - a promising DNA vaccine delivery system. Med Sci 6:27. https:// doi.org/10.3390/medsci6020027

84. Zhang Y, Zeng G, Pan H et al (2021) Safety, tolerability, and immunogenicity of an inactivated SARS-CoV-2 vaccine in healthy adults aged 18-59years: a randomised, double-blind, placebocontrolled, phase 1/2 clinical trial. Lancet Infect Dis 21:181-192. https://doi.org/10.1016/S14733099(20)30843-4

85. Zhu FC, Guan XH, LiYHetal (2020) Immunogenicity and safety of a recombinant adenovirus type5 -vectored COVID-19 vaccine in healthy adults aged 18 years or older: a randomised, doubleblind, placebo-controlled, phase 2 trial. Lancet 396:479-488. https://doi.org/10.1016/S0140 6736(20)31605-6

86. Zrzavy T, Kollaritsch H, Rommer PS et al (2019) Vaccination in multiple sclerosis: friend or foe? Front Immunol. https://doi.org/10.3389/fimmu. 2019.01883

87. https://clinicaltrials.gov/ct2/show/NCT04510207. Zugegriffen: 05.04.2021

88. https://clinicaltrials.gov/ct2/show/NCT04526990. Zugegriffen: 22.01 .2021

89. https://covid19.trackvaccines.org/vaccines/. Zugegriffen: 23.04 .2021

90. https://covid19.who.int/.Zugegriffen:24.04.2021

91. https://www.Ema.Europa.Eu/En/Documents/ Product-Information/Covid-19-VaccineAstrazeneca-Product-Information-ApprovedChmp-29-January-2021-Pending-Endorsement En.Pdf.Zugegriffen:29.01.2021

92. https://www.Ema.Europa.Eu/En/Documents/ Product-Information/Mavenclad-Epar-ProductInformation_En.Pdf. https://www.ema.europa. eu/en/documents/product-information/ mavenclad-epar-product-information_en. pdf.Zugegriffen:01.04.2021

93. https://www.ema.europa.eu/en/news/astrazenecas covid-19-vaccine-ema-finds-possible-link-veryrare-cases-unusual-blood-clots-low-blood. Zugegriffen: 07.04.2021

94. https://www.ema.europa.eu/en/news/emarecommends-covid-19-vaccine-janssenauthorisation-eu.Zugegriffen: 11.03.2021

95. https://www.ema.europa.eu/en/news/firstcovid-19-vaccine-safety-update-published. Zugegriffen: 29.01.2021

96. https://Ec.Europa.Eu/Commission/Presscorner/ Detail/De/Fs_21_650.Zugegriffen:24.04.2021

97. https://www.fda.gov/emergency-preparednessand-response/coronavirus-disease-2019-covid19/janssen-covid-19-vaccine. Zugegriffen: 23.04.2021

98. https://www.fda.gov/news-events/pressannouncements/fda-and-cdc-lift-recommendedpause-johnson-johnson-janssen-covid-19vaccine-use-following-thorough. Zugegriffen: 23.04.2021

99. https://gth-online.org/wp-content/uploads/ 2021/04/GTH-Stellungnahme-AstraZeneca_41-2021.pdf.Zugegriffen:01.04.2021
100. https://www.kompetenznetz-multiplesklerose. de/.Zugegriffen:01.03.2021

101. https://www.msif.org/news/2020/02/10/thecoronavirus-and-ms-what-you-need-to-know/. Zugegriffen: 23.04.2021

102. https://www.mssociety.org.uk/what-we-do/ news/ms-society-medical-advisers-releaseconsensus-statement-covid-19-vaccines. Zugegriffen: 12.03.2021

103. https://www.nationalmssociety.org/coronaviruscovid-19-information/multiple-sclerosisand-coronavirus/covid-19-vaccine-guidance/ Timing-MS-Medications-with-COVID-19-mRNAVaccines.Zugegriffen: 12.03.2021

104. https://www.oegn.at/covid-19/covid19-undmultiple-sklerose-ms/.Zugegriffen:01.03.2021

105. https://www.pei.de/DE/newsroom/hp-meldungen/ 2021/210322-auftreten-hirnvenenthrombosennach-impfung-covid-19-impfstoff-astrazeneca. html.Zugegriffen:22.03.2021

106. https://www.rki.de/DE/Content/Kommissionen/ STIKO/Empfehlungen/Stellungnahme-Impfabstand. html\#: :text=Die\%20STIKO\%20empfiehlt\%2C $\% 20$ die $\% 20$ Impfung, dem\%20AstraZeneca \%2DImpfstoff\%20zu\%20verabreichen. Zugegriffen: 14.04 .2021

107. https://www.sozialministerium.at/Themen/ Gesundheit/Impfen/Impfplan-\%C3\%96sterreich. html.Zugegriffen:01.04.2021

108. https://www.who.int/publications/m/item/draftlandscape-of-covid-19-candidate-vaccinestle. Zugegriffen: 23.04 .2021 